\title{
Solid-state block-based pulsed laser illuminator for single-photon avalanche diode detection-based time-of-flight 3D range imaging
}

\author{
Sahba S. Jahromi $\odot$, Jussi-Pekka Jansson, ${ }^{a}$ Pekka Keränen $\odot$, \\ Eugene A. Avrutin, ${ }^{\mathrm{b}}$ Boris S. Ryvkin, ${ }^{\mathrm{a}, \mathrm{c}}$ and Juha T. Kostamovaara $\odot^{\mathrm{a}, *}$ \\ ${ }^{a}$ University of Oulu, Circuits and Systems Research Unit, ITEE Faculty, Finland \\ ${ }^{b}$ University of York, Department of Electronic Engineering, England, United Kingdom \\ ${ }^{c}$ A.F. Ioffe Physico-Technical Institute, St. Petersburg, Russia
}

\begin{abstract}
A laser diode illuminator for single-photon avalanche diode detection-based pulsed time-of-flight 3D range imaging is presented. The illuminator supports a block-based illumination scheme and consists of a 16-element custom-designed common anode quantum-well laser diode bar working in the enhanced gain switching regime and lasing at $\sim 810 \mathrm{~nm}$. The laser diode elements are separately addressable and driven with gallium nitride drivers, which produce current pulses with a width of $\sim 2 \mathrm{~ns}$; the current pulse amplitude was estimated from the supply voltage $(90 \mathrm{~V})$ as 5 to $10 \mathrm{~A}$. Cylindrical optics are used to produce a total illumination field-ofview of $40 \times 10 \mathrm{deg}^{2}$ (full width at half maximum) in 16 separately addressable blocks. With a laser pulsing frequency of $256 \mathrm{kHz}$ and laser pulse energy of $\sim 8.5 \mathrm{~nJ}$, the average optical illumination power of the transmitter is $2.2 \mathrm{~mW}$. () The Authors. Published by SPIE under a Creative Commons Attribution 4.0 Unported License. Distribution or reproduction of this work in whole or in part requires full attribution of the original publication, including its DOI. [DOI: 10.1117/1.OE.60.5.054105]
\end{abstract}

Keywords: laser radar; range imaging; lidar; single-photon detection.

Paper 20210219 received Mar. 1, 2021; accepted for publication May 4, 2021; published online May 21, 2021.

\section{Introduction}

3D range imaging has traditionally been used in surveying, civil engineering, inspection, and quality control, ${ }^{1,2}$ but there has been a growing interest in exploiting these techniques in a wider field of applications, e.g., for environment sensing in autonomous cars. Other relevant applications could be robotics, security, small vehicle guidance, e.g., Unmanned Aerial Vehicles, virtual reality/augmented reality, consumer electronics (games), and man-machine interfaces, e.g., gesture control. ${ }^{3-5}$

One potential alternative for 3D range imaging is the pulsed time-of-flight (TOF) approach using CMOS single-photon avalanche detector (SPAD) techniques, in which a burst of short, powerful optical pulses produced with a laser diode transmitter is used to illuminate the field-ofview (FOV) of the system. The receiver consists of a 2D array of SPADs located approximately at the focal plane of the receiver optics, as shown in Fig. 1.6,7

In this system, some of the transmitted photons will be reflected from the target and hit the CMOS 2D single-photon receiver array. A photon hitting the active area of any of the singlephoton detectors may then be detected as a result of the high-speed breakdown in the detector. The timing jitter of a CMOS single-photon detection is of the order of only $50 \ldots 100 \mathrm{ps}^{8}{ }^{8}$ The breakdown immediately introduces a logic-level signal (e.g., $3 \mathrm{~V}$ ) and thus no analog amplifiers are needed. In addition to the 2D array of SPAD detectors, the receiver also includes an array of time-to-digital converters (TDCs), the function of which is to measure the interval between the emitted laser pulse and the breakdown introduced into each SPAD pixel that has detected a photon. These intervals represent the transit times $(\Delta t)$ of the photons from the transmitter to the target and back to the receiver array. Since every pixel in the array only sees a small

*Address all correspondence to Juha T. Kostamovaara, juha.kostamovaara@oulu.fi 


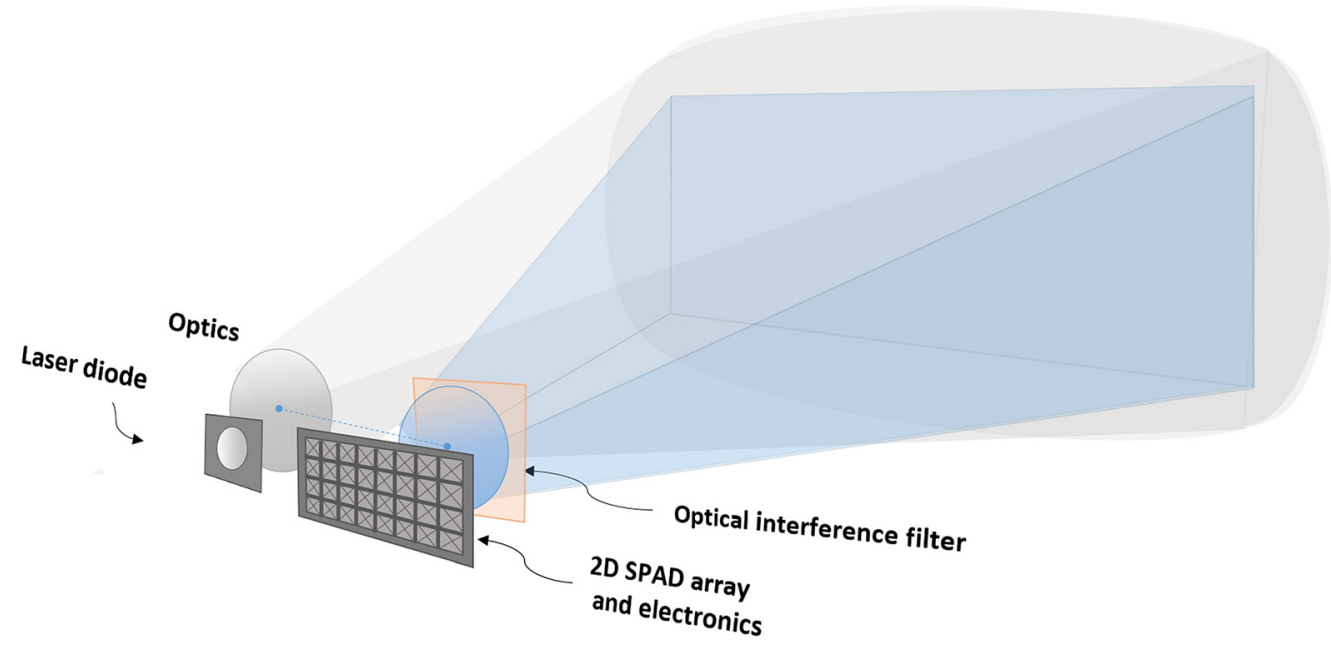

Fig. 1 Basic functionalities of the SPAD receiver-based solid-state pulsed TOF 3D range imager concept.

portion of the FOV of the receiver, as defined by its relative position in the array (the receiver array is approximately at the focal plane of the receiver optics), a 3D range image can be achieved in a solid-state device implementation. In this 3D range image, the $x$ and $y$ coordinates are uniquely determined from the location of the pixel in the array, whereas the $z$ coordinate by is recovered from the measured transit time $[z=R=c *(\Delta t / 2)]$ of the photon introducing the breakdown in the corresponding pixel.

One specific practical feature of this measurement technique is that, in the case of a typical measurement, the probability of a single SPAD element detecting a signal photon is substantially below one within most of the measurement range. This means that it is necessary to transmit a number of optical pulses to achieve a valid detection for each pixel, i.e., a high enough signal-tonoise ratio (SNR). Thus, in the measurement sequence of a 3D image, a bunch of individual laser pulses (e.g., 1000 pulses) is transmitted to produce a histogram of single-shot results for each pixel element $(x, y)$ since each individual emitted pulse has only a very low detection probability. The "noise" in the histogram is predominantly produced by random background hits, and the "signal" by the signal hits occurring at a time interval corresponding to the transit time of the photons to the target and back to the receiver. ${ }^{9-13}$

It is well known that focusing of the available average optical illumination power into energetic ns or even sub-ns impulse-like laser pulses improves the precision of the system and its SNR, especially under high background illumination conditions. ${ }^{13}$

Many recent developments within this field have focused on the development of high performance, versatile 2D SPAD receivers with on-chip TDCs and signal processing units supporting a flood illumination-based flash 3D LIDAR architecture (as in Fig. 1). ${ }^{14-18}$ Recently, however, it has been proposed that better system performance, within the limits of the permitted average illumination power, could be achieved with solid-state block-based illumination. ${ }^{13,19-21}$ This is a strategy, in which only part of the system FOV is illuminated by each emitted laser pulse, e.g., a narrow block covering $1 / 16^{\text {th }}$ of the total FOV of the system (marked in yellow in Fig. 2). The receiver would then consist of a SPAD array covering the whole system FOV, but since only $1 / 16^{\text {th }}$ of the FOV is being illuminated per emitted laser pulse, only the corresponding set of receiver SPAD elements (the yellow block in the receiver in Fig. 2, 2 lines of 128 elements in the receiver array) are activated and connected to the TDC bank. For the next laser pulse (or bunch of laser pulses), the second sub-FOV (second blue in Fig. 2) is illuminated and the corresponding SPADs in the receiver array are activated. The operation then continues following the same algorithm.

Since it is assumed that all the available energy of the transmitter (which is typically limited by eye safety or laser diode driver capacity) is used to illuminate the selected sub-FOV, blockbased and homogenous forms of illumination are equivalent from the signal point of view. In other words, in block-based illumination a single SPAD element is illuminated only by $1 / 16^{\text {th }}$ of 


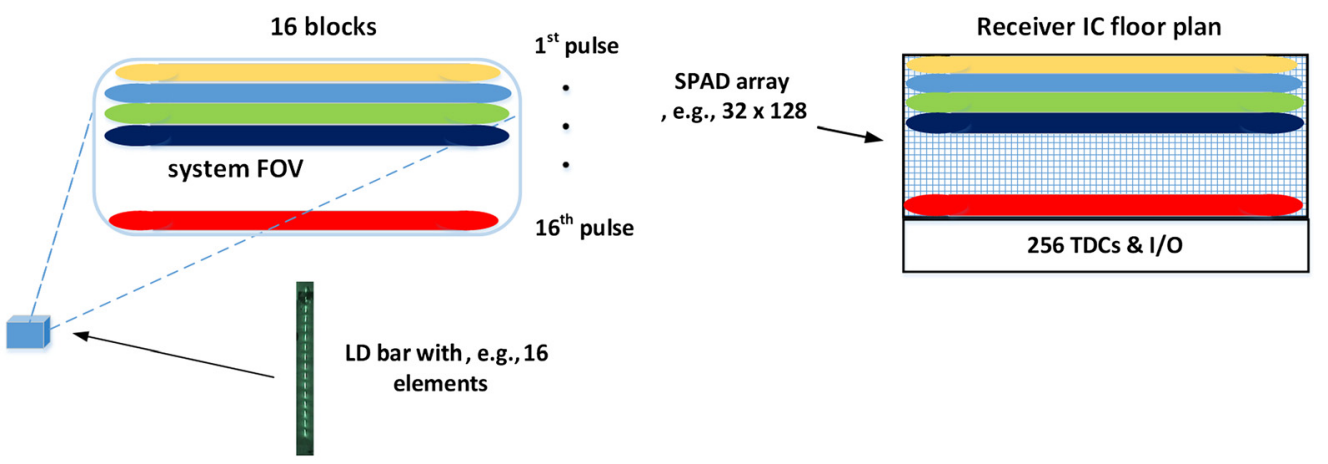

Fig. 2 Block-based illumination: concept, transmitter, and receiver.

the total number of laser pulses in a given frame period, as shown in Fig. 2. However, the energy per active pixel and per laser pulse is 16 times higher (and thus the probability of a photon detection in a SPAD pixel is 16 times greater). As a result, the number of signal detections in a given measurement time (defined by the desired frame rate) is exactly the same as it would be when using flood illumination with the same amount of average optical energy (assuming operation in single-photon detection regime, which holds for practical measurement cases).

However, from the point of view of noise, the situation is different. Since each SPAD is active for $1 / 16^{\text {th }}$ of the number of laser pulses used for the total 3D frame cycle, the total amount of noise detections is also reduced by a factor of 16 . This follows from the fact that the noise is proportional to the ratio of the length of the detection window (which is 16 times shorter than with flood illumination) to the mean time interval between random detections (caused by detector dark counts and the background illumination, which typically dominates in outdoor measurements). As a result, the SNR (which is proportional to the ratio of the number of signal detections to the square root of the noise detections), would then be four times $[\sqrt{ }$ (number of blocks) $]^{13}$ better, which means that a lower number of laser pulses would be needed to achieve the same SNR as with flood illumination. This improved SNR can be used to speed up the measurements (by a factor related to the number of blocks) or to increase the maximum measurement range. ${ }^{13,19}$

An important additional advantage is that the number of TDCs needed would be much lower with block-based illumination than with flood illumination (by a factor of 16 in the above example). This is a significant advantage since it markedly lowers the complexity and costs of the receiver circuit. In addition, the illumination can now be adaptive in the sense that it can be focused selectively on precisely that part of the total system FOV that contains interesting information. Thus, a smaller field of view can be used in vehicle applications, for example, to increase the SNR within a certain frame update rate for long ranges whereas for a short range the illuminated FOV can be increased (trading spatial resolution for FOV).

Thus, it is in general advantageous in SPAD-based pulsed TOF 3D ranging to focus the pulse energy in terms of time and space. This approach, however, while reducing the complexity of the receiver, would obviously increase that of the transmitter. In principle, the solid-state transmitter could be realized using either a two-dimensional vertical-cavity surface-emitting laser (VCSEL) array with separately addressable unit elements or a multi-element edge-emitting laser diode bar with multiple parallel emitters. The advantage of addressable VCSEL array techniques is the relatively simple realization of the optics since the dimensions of the VCSEL array and its elements can be scaled to correspond to the desired illumination pattern. On the other hand, it is hard to go to an ns (and especially sub-ns) high-energy pulse regime with VCSELs, which would in turn detract from the precision of the measurements. The other obvious realization option would be the laser diode bar approach, in which cylindrical optics could be used to produce vertical or horizontal illumination blocks.

This paper presents what we believe as the first published solid-state realization of a blockbased optical illuminator intended for SPAD-based pulsed TOF 3D range imaging. The transmitter consists of a custom-designed common anode laser diode (LD) bar with 16 elements, each following the "enhanced gain switching" design principle that allows for high energy $(\sim \mathrm{nJ})$ sub-ns pulse production with a relatively simple driving scheme. ${ }^{22}$ The illuminator optics 
produce a FOV of $\sim 40 \times 10 \mathrm{deg}^{2}$ and the whole illuminator is realized on a single small-sized printed circuit board.

The rest of the paper is organized as follows: the design of the laser diode bar will be briefly described in Sec. 2, Sec. 3 will present the design principles of the laser diode driver electronics and optics, and results arising from characterization of the designed transmitter will be given in Sec. 4. The results will be discussed and summarized in Sec. 5.

\section{Emitter Design}

The laser emitter used here is an LD bar with 16 individual emitting elements. The design of individual lasers in the bar is tailored for the emission of short, intense pulses under an enhanced gain-switched operation regime. ${ }^{22-24}$ More specifically, the relatively short resonator length of $1.5 \mathrm{~mm}$ contributes to a relatively short gain-switching pulse duration (of $\sim 70 \mathrm{ps}$ ), whereas the broad stripe width of $150 \mu \mathrm{m}$ helps ensure high overall pulse energy. The laser waveguide (similar to that used in Ref. 24) is a broad asymmetric structure ensuring a single transverse mode of operation with a broad near field. The active layer of each laser consists of five GaAs/AlGaAs Quantum Wells, each $40 \AA$ thick, with a total thickness of the active material of $d_{a} \sim 200 \AA$. The position of the active layer is shifted toward the $p$-cladding, making for a relatively large equivalent spot size, $d_{a} / \Gamma_{a}>\sim 3 \mu \mathrm{m}$. As shown in previous papers, ${ }^{22-24}$ a high value for $d_{a} / \Gamma_{a}$ enables the accumulation of a large number of carriers in the active layer before the emission of the gain-switching pulse, leading to high gain-switching pulse energy (enhanced gain switching). Such a regime is also characterized over a broad range of current pulse amplitudes by the optical pulse being emitted near the trailing edge of the current pulse, making for a single optical pulse, free of any after-pulsing structure. Single optical pulse energies of up to $\sim 1 \mathrm{~nJ}$ have been demonstrated in QW lasers, ${ }^{24}$ and even higher pulse energies can be obtained if an after-pulsing structure is acceptable for device operation, as in the current study.

\section{Laser Diode Bar Driver Electronics}

As already mentioned, it is advantageous in SPAD-based pulsed TOF ranging to focus the available average power into short, energetic optical pulses. Assuming a slope efficiency of a single junction laser diode of $\sim 1 \mathrm{~W} / \mathrm{A}$, an optical pulse energy of $\sim 5 \mathrm{~nJ}$, and a pulse duration length of $\sim 1 \mathrm{~ns}$ would require a drive current pulse of $\sim 5 \mathrm{~A} / 1 \mathrm{~ns}$ for the laser diode, assuming that the optical output follows the current adiabatically. In principle, the laser diode could be driven with a high-speed nMOS switch. The realization of drive electronics permitting the above parameters or ones that are even more demanding is not a straightforward matter, however. Since the slew-rate of the current rise is limited by the loop inductance, it is important to minimize this. Assuming, for example, a loop inductance of just $5 \mathrm{nH}$, the slew-rate of a current with a supply voltage of $50 \mathrm{~V}$ would be $10 \mathrm{~A} / \mathrm{ns}$. The effect of the inductance can be suppressed by a series resistance (since $\tau=L / R$ ), but this obviously would limit the maximum current (or require a higher supply voltage) as well.

Another issue is the voltage transient induced at the drain of the nMOS switch at the trailing edge of the current pulse (switch-off situation) due to the accumulated loop inductance energy. This may damage the switch and should thus be minimized.

In this design, the laser diode bar was realized as a custom common anode structure since this allows each of the laser diode elements to be separately driven with a high-speed gallium nitride $(\mathrm{GaN})$ FET transistor with the driver electronics simplified considerably (see Fig. 3 for a schematic diagram of the laser transmitter electronics). The pulsing sequence is produced with an FPGA triggered at $256 \mathrm{kHz}$. This FPGA triggers the GaN drivers sequentially, providing pulses of $5 \mathrm{~ns}$ to the gates of the $\mathrm{GaN}$ switches so that the pulsing rate of each of the laser diodes is $16 \mathrm{kHz}$.

In the driver configuration used here, the current pulse length is defined by the LC transient of the laser diode loop $\left(\Delta T \sim 2 \sqrt{ } L_{\text {loop }} C_{L}\right)$. This transient is damped by the resistor $R_{D}$ $\left(\sim \sqrt{ }\left(L_{\text {loop }} / C_{\mathrm{L}}\right)\right)$, and thus the maximum current amplitude is roughly $V_{H V} /\left(\left(\sqrt{ }\left(L_{\text {loop }} /\right.\right.\right.$ $\left.\left.C_{L}\right)+R_{D}\right) \cdot{ }^{25,26}$ 


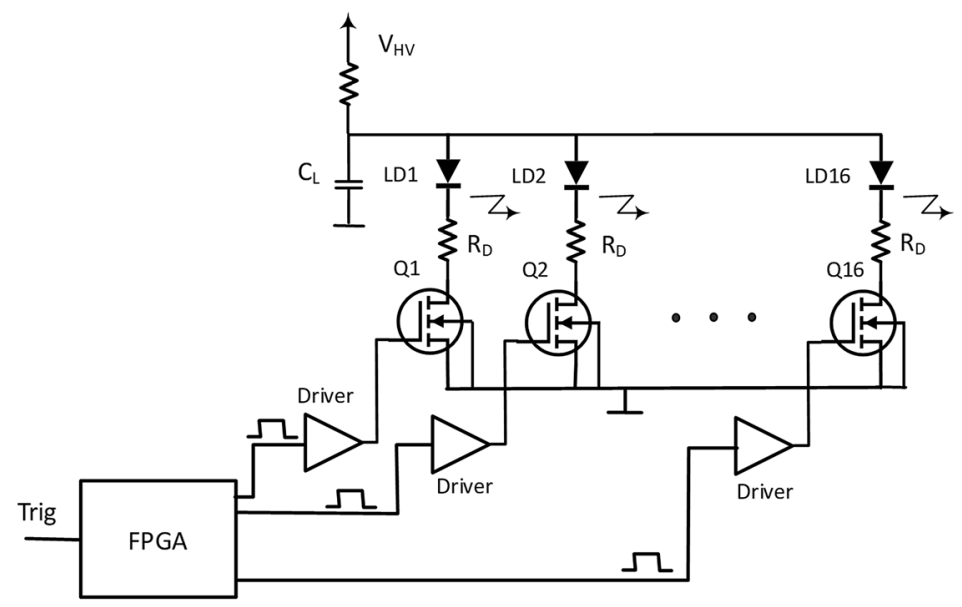

Fig. 3 Schematic diagram of the 16-element laser diode bar transmitter electronics.

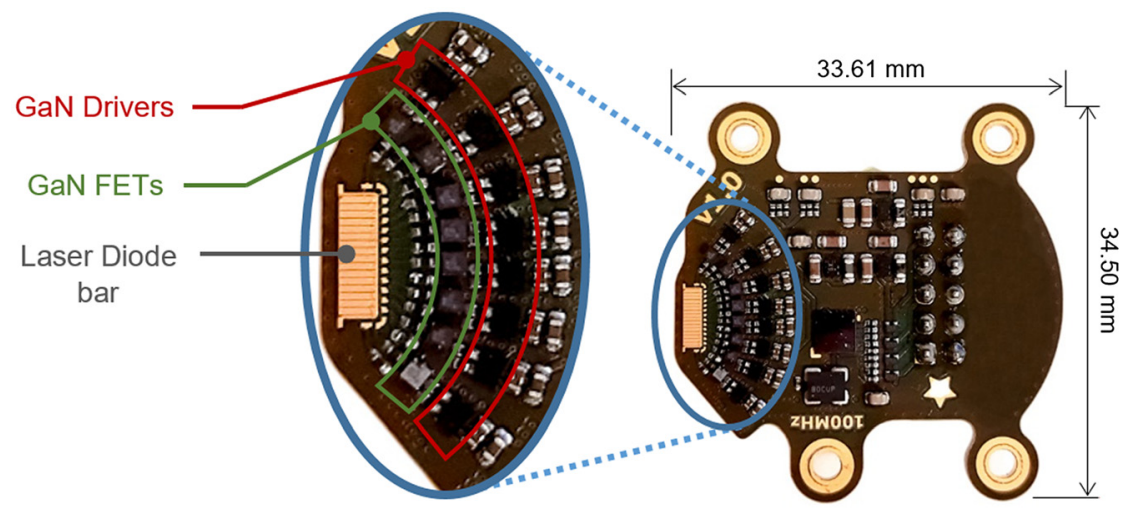

Fig. 4 Printed circuit board realization of the 16-element laser diode bar transmitter.

In the PCB realization of the 16-element laser diode bar transmitter shown in Fig. 4, the discharging capacitor $C_{L}$ has a capacitance value of $100 \mathrm{pF}$, the loop inductance (not shown in the schematics) is estimated to be 5 to $8 \mathrm{nH}$ (depending on the position of the LD in the bar) and the damping resistor $R_{D}$ is set to $5 \Omega$. Thus, a peak amplitude of $\sim 6 \ldots 7 \mathrm{~A}$ and pulse width of $\sim 2 \mathrm{~ns}$ [full width at half maximum (FWHM)] can be expected for a laser diode drive current pulse with a supply voltage $V_{H V}$ of $90 \mathrm{~V}$.

It should be noted that since the laser diodes are working in the enhanced gain switching mode, the optical output consists of a short high-power main pulse ( $100 \mathrm{ps} \mathrm{FWHM,} \mathrm{an} \mathrm{order} \mathrm{of}$ magnitude shorter than the current pulse duration), followed by an afterpulsing tail structure at a much lower power level (more detail in Sec. 4). Thus, the use of the enhanced gain switching technique significantly relaxes the requirements put on the driver electronics regarding the duration of the generated current pulse.

The pitch of the laser diode bar is $300 \mu \mathrm{m}(150-\mu \mathrm{m}$ active stripe width, $150-\mu \mathrm{m}$ separation). Cylindrical optics are used to convert the output of the laser diode bar into an illumination cone with a total FOV of $40 \times 10 \mathrm{deg}^{2}$ (FWHM). When focused at infinity, 16 illumination stripes (or blocks) with a separation corresponding to the width of a single line are produced, and the illumination pattern can be made more homogenous by slightly misfocusing the transmitter. This is demonstrated in Fig. 5, which shows simulated illumination patterns produced by the optics designed here from an LD bar with the above parameters. These patterns are simulated at a distance of $10 \mathrm{~m}$, the scales on the $x$ and $y$ axes being in millimeters. The homogenizing effect of the misfocus is clearly seen [Fig. 5(b)]. 


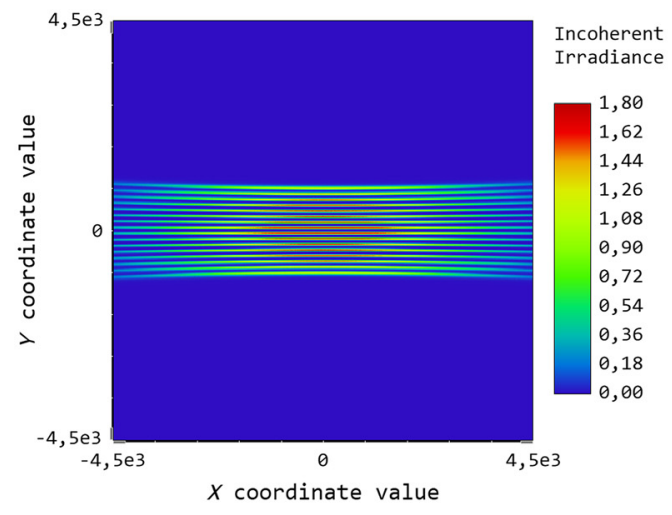

(a)

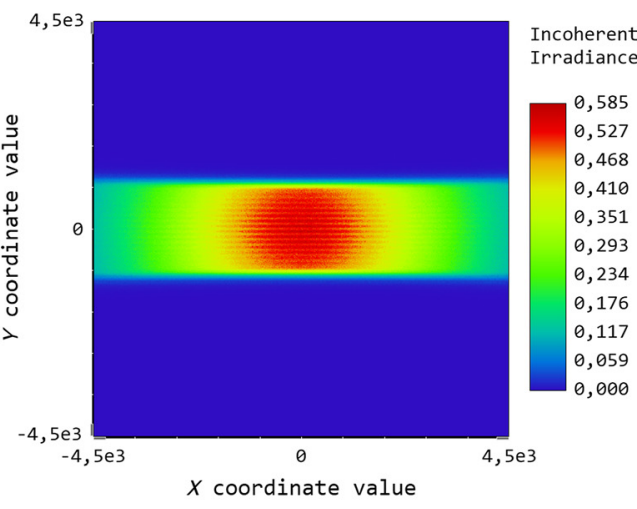

(b)

Fig. 5 (a) Simulated illumination patterns for focused and (b) misfocused 16-element transmitters.

\section{Characterization of the Block-Based Illuminator}

The optical output from the laser diode bar was characterized with a bandwidth of $\sim 25 \mathrm{GHz}$ (eqv. rise time $\sim 14$ ps, New Focus, high-speed optical probe 1434, and Tektronix mixed-signal oscilloscope 73304DX). Typical results are shown in Fig. 6. Green and red curves show the measured average pulse shapes for the individual laser diode elements with the maximum $(\sim 9.8 \mathrm{~nJ})$ and minimum energy ( $\sim 6.8 \mathrm{~nJ})$ total pulse energy among the lasers in the bar, respectively. The black curve shows the average pulse shape for all 16 laser diode elements. The gain switching peak in the leading part of the pulse has an average FWHM width of $\sim 70$ ps (as measured from the recorded laser diode outputs shown in Fig. 6) and pulse energy of 1.5 to $3 \mathrm{~nJ}$, which is considerably higher than what is available with standard gain switching techniques. ${ }^{27,28}$ The lasers have been designed to give the center wavelength of the laser radiation of $\sim 810 \mathrm{~nm}$.

A photograph of the measured illumination pattern in a focused measurement situation at a target distance of $\sim 1 \mathrm{~m}$ is shown in Fig. 7. It is worth noting that although all the lines seem to be illuminated (due to the long exposure time), in reality only one of the laser diode elements is being driven at any given time.

The measured detection probability of the pixels under a single illuminated line (LD element 8 ) is shown in Fig. 8. This measurement was carried out at a distance of $\sim 10.5 \mathrm{~m}$ from the target, a painted wall with an estimated reflection coefficient of $\sim 1$ (equivalent to white paper). The result was recorded with the SPAD receiver described in more detail in Ref. 19. This receiver consists of a single CMOS chip of $32 \times 128$ SPAD pixels and $2 \times 128$ TDC elements that can be

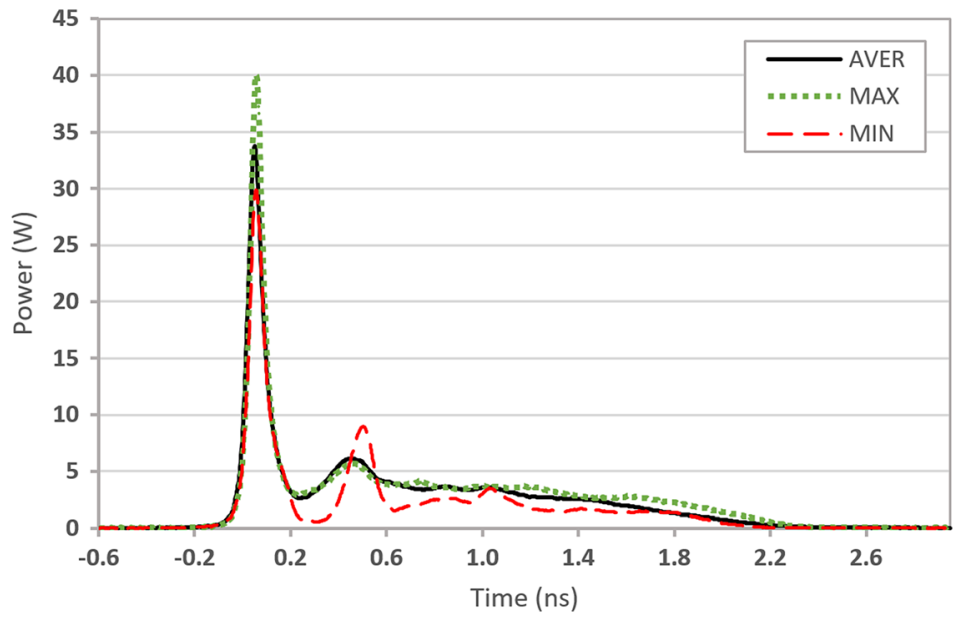

Fig. 6 Time-domain optical responses of two LD bar elements (green and red) and the averaged optical output of all 16 laser diode elements (black). 


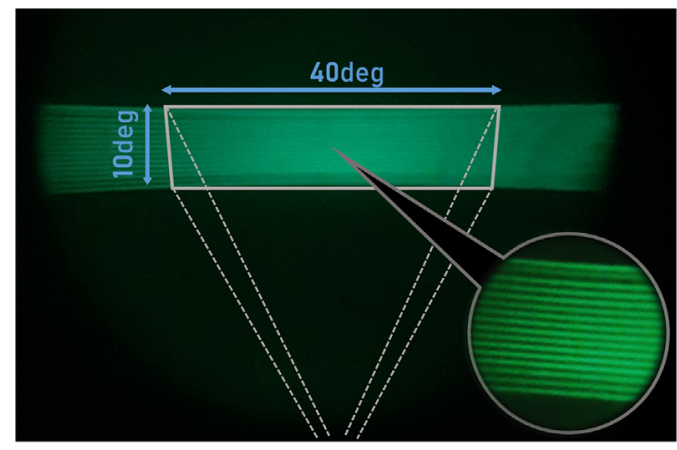

Fig. 7 Photograph of the illumination pattern at a distance of $\sim 1 \mathrm{~m}$.

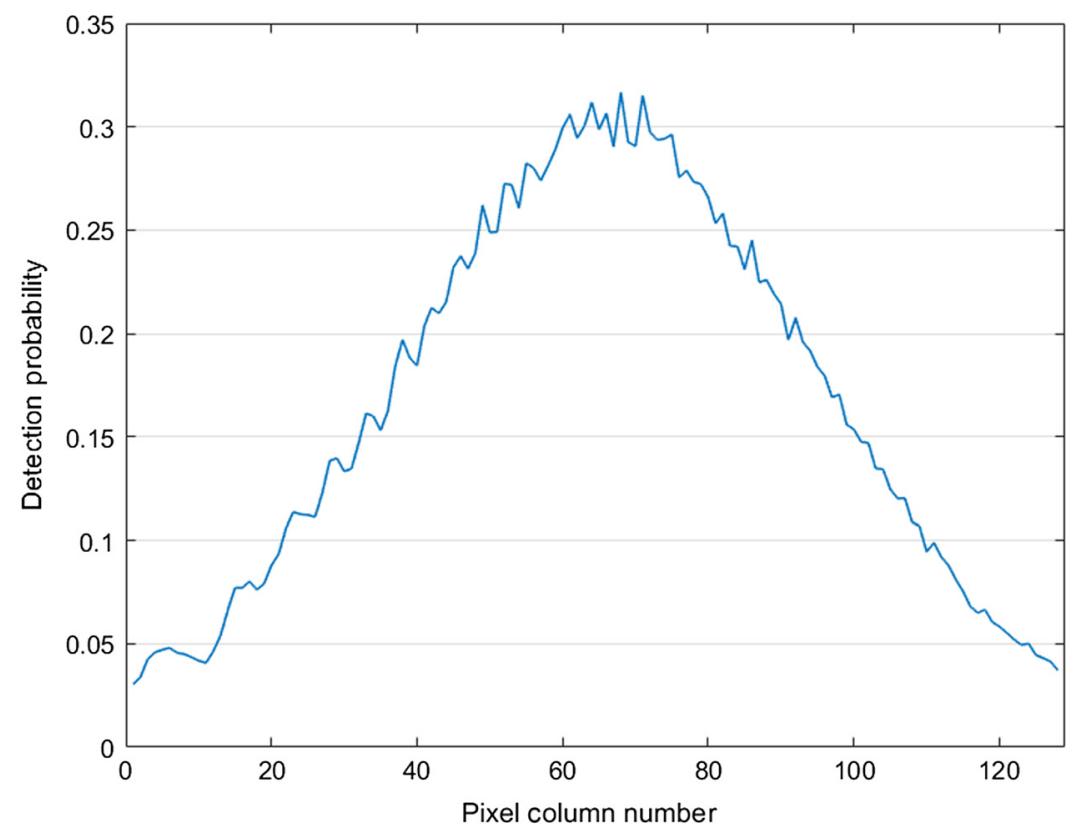

Fig. 8 Detection probabilities of 128 SPAD pixels along a single SPAD receiver line. The combined width of the 128 SPAD pixels corresponds to a FOV of 40 deg.

electrically connected to any two lines within the SPAD arrays. The image of the transmitted beam (focused at infinity) was focused onto the array of $32 \times 128$ SPAD detectors using receiver optics with a focal length of $6.67 \mathrm{~mm}$ and an aperture of $5.6 \mathrm{~mm}$. The detection probability is proportional to the intensity of the received optical energy in a horizontal direction. The total pulse energy was included in the calculation of the detection probability by summing all the hits within the envelope of the detection histogram (full width $=\sim 3 \mathrm{~ns}$ ) for each pixel.

As can be seen, the intensity of the received optical field declines at the edges of the FOV of the system (FWHM $\sim 25 \mathrm{deg}$ ), this effect being stronger than could be expected from the variation in the intensities of the transmitter illumination patterns (Fig. 5). The additional reduction is attributed to the cosine effect of the receiver optics. It is evident that the detection probability for all the pixels is $<1$.

The detection probability for the whole receiver is also shown in color-coded form in Fig. 9, indicating that in the focused situation the detection rate in the SPAD lines next to the one having the highest intensity is markedly lower. This can be expected since the transmitter-receiver optics were designed to project an image of the $150-\mu \mathrm{m}$ wide laser stripe onto the top of the $40-\mu \mathrm{m}$ wide SPAD line. Thus, the detection probability in every second line of the $32 \times 128$ SPAD array would be lower, but, as indicated above, the difference could be smoothed out by slightly misfocusing the transmitter. 


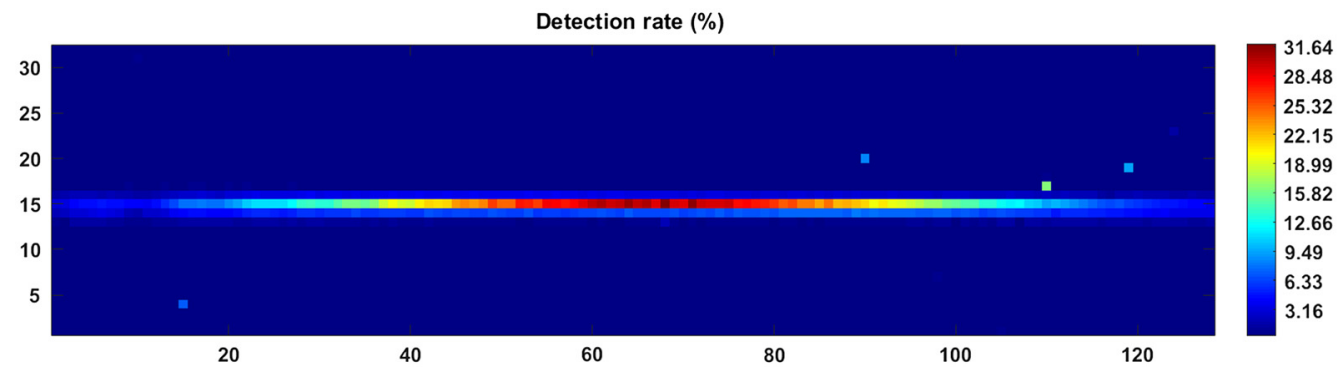

Fig. 9 Detection probability in the $32 \times 128$ SPAD receiver array under focused measurement conditions. A single element within the 16-element laser diode bar was used to illuminate a target located at $10.5 \mathrm{~m}$.
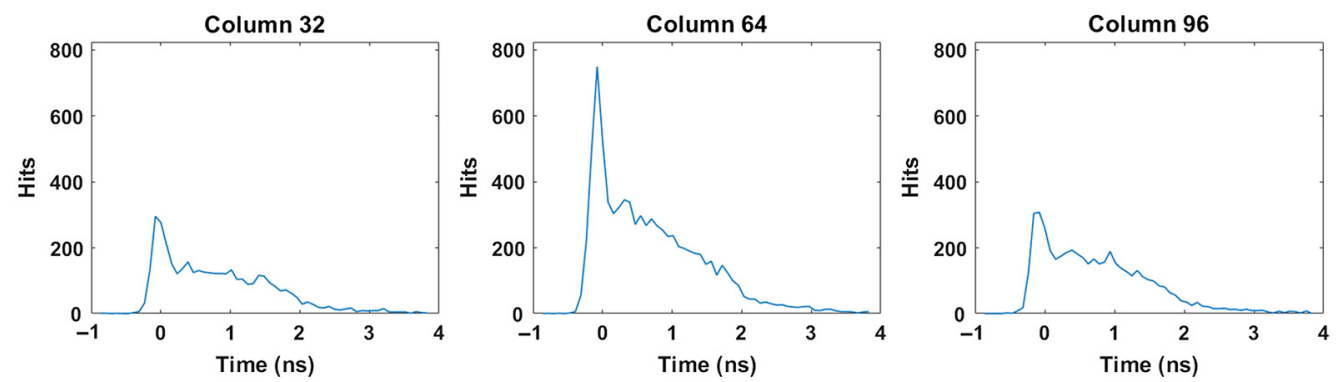

Fig. 10 Time histograms of detections from a target located at $10.5 \mathrm{~m}$ in single SPAD detectors. 25,600 laser shots were used to assemble the histogram. The time positions of the histograms are arbitrary for the sake of clarity. TDC LSB $\sim 80 \mathrm{ps.}$

As mentioned above, the CMOS receiver includes also a bank of TDCs, which can measure the time position of a SPAD detection in relation to the emitted laser pulse at a resolution of $78 \mathrm{ps}$. Some sample raw histograms of hits from a target (white wall) at a distance of $10.5 \mathrm{~m}$ received by three detectors located in row 15 (max. illumination) and columns 32, 64, and 96, respectively, are shown in Fig. 10. A total of 25,600 laser pulses were used to assemble these histograms.

It is seen that the widths of the gain switching peaks of the histograms are considerably wider than in the optical pulses shown in Fig. 6 ( $\sim 70 \mathrm{ps}$ ), measuring $\sim 250 \ldots 350$ ps depending on the position of the recording SPAD in the array. Another notable feature is the reduction in the ratio of the level of the gain-switching peak to that of the tail part of the distribution toward the edge of the FOV. These effects are caused by the transit time spread of photons within the pixel, which is larger at the edge of the FOV, and partly by the system jitter. The dependence of the laser pulse shape on the far-field angle direction may also have some effect on this change.

The average detection probability within the $40 \mathrm{deg}$ system FOV is $\sim 0.15$, which agrees very well to radiometric calculations using the $8.5 \mathrm{~nJ}$ total pulse energy for this particular laser diode element. If, for example, a laser pulsing rate of $256 \mathrm{kHz}$ were to be used, the above result suggests that for such a set-up a measurement range of $>30 \mathrm{~m}$ could be achieved under low background illumination conditions (1 klux) at a frame rate of 10 frames/s. In this case, the average optical illumination power of the transmitter would be $2.2 \mathrm{~mW}$. Under more demanding background conditions, the SNR, and thus the maximum range, would obviously be lower and design strategies would be needed to counteract the blocking effect of background detections. ${ }^{9-13}$

Figure 11 shows a 3D range point cloud image created based on the above results, i.e., with 25,600 measurements performed for each pixel while only one of the LD elements was active in the 16-element laser diode bar, the target being a flat white wall located at a distance of $10.5 \mathrm{~m}$ from the 3D imagers. Histograms of the hits were gathered for each pixel, and cross-correlation with a filter is used to translate the histograms into distances. The concerned filter was a normalized version of a histogram of hits collected by the middle SPAD measuring the location of a target placed at a specific distance from the camera. It can be seen that although only one laser diode element in the 16-element bar was used in this experiment, a reliable 3D range image could 


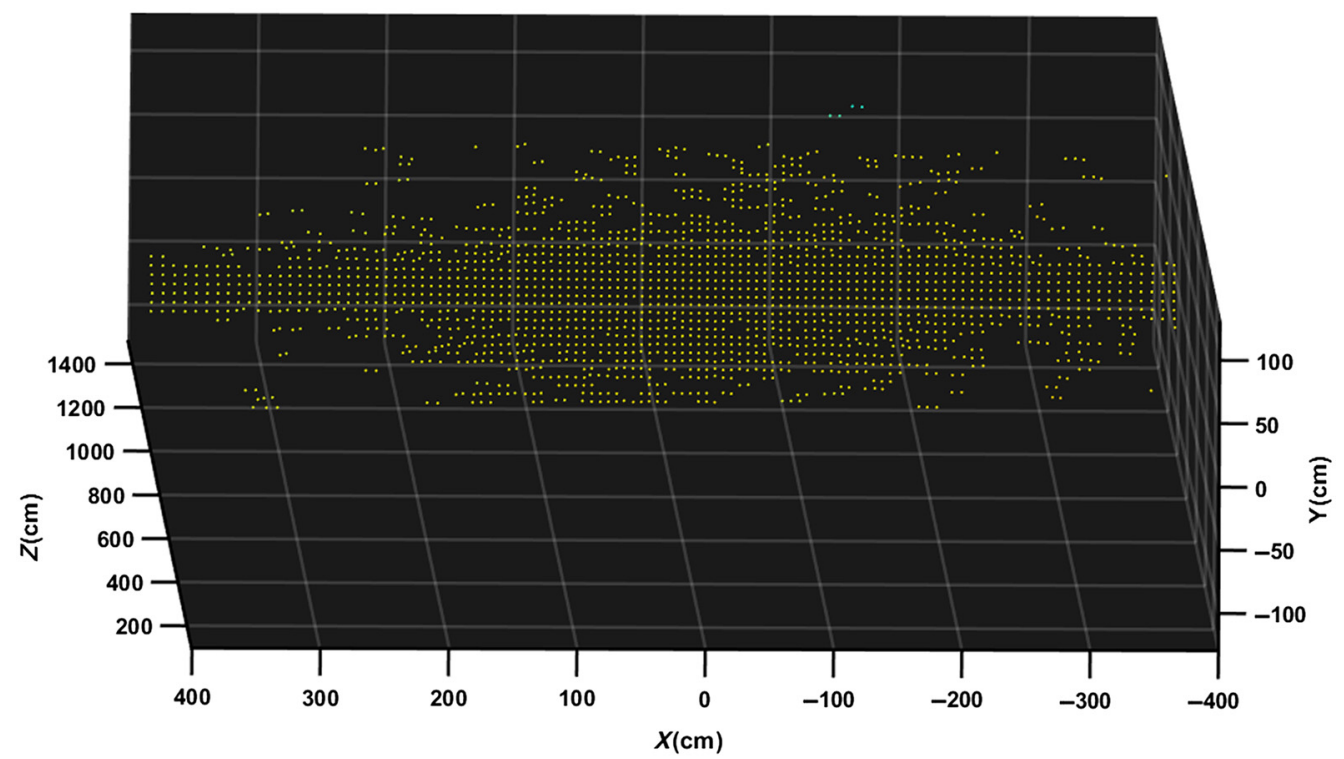

Fig. $113 \mathrm{D}$ point cloud image of a flat white wall located at $10.5 \mathrm{~m}$, created using the illumination from one LD.

be produced using several other receiver array lines with 128 SPAD pixels (5 . 6 lines). Some results could also be achieved outside this area.

\section{Discussion and Summary}

The key result of this work is a practical demonstration of a solid-state (i.e., fully electronic) block-based illuminator realized relatively straightforwardly and in miniature size with a common anode laser diode bar and cylindrical optics. The common anode structure enables the use of n-type enhancement GaN field-effect transistors to drive the laser diode elements with pulse currents in a $\sim 10 \mathrm{~A} / \sim 1 \mathrm{~ns}$ regime, which is important for optimizing the SNR of a SPAD-based pulsed TOF laser range 3D imager. With a specially designed laser diode working in the enhanced gain-switching mode, the optical pulse can be suppressed even to sub-ns duration, which could be beneficial when aiming at mm-range precision. To increase the measurement range, especially under difficult background conditions, the pulse energy (and pulsing rate) should obviously be increased from the values used here. It should be noted also that the design principles shown here can equally well be applied to laser diodes working in the standard pulse regime while allowing for the higher pulse energy, albeit at the cost of a longer pulse and hence more coarse resolution.

The proposed approach allows, in principle, two operation modes with regard to illumination. In one mode, the active stripes of the separate elements in the laser diode bar are focused to fit onto a single SPAD detector line in the 2D detector array (as in the above measurements, e.g., in Fig. 11). Using this mode, it is actually the transmitter that would determine the spatial resolution in the direction of the illumination line (the number of laser diode elements), especially at the maximum range, whereas the spatial direction in the perpendicular direction would be defined by the number of SPAD pixels in each line. The advantage of this approach is the high degree of spatial focusing, which is known to maximize the SNR in the measurements, especially under conditions of high background illumination. It is also noted that the full resolution of the $2 \mathrm{D}$ receiver array can be used for shorter distances due to the transmitted energy, which falls in between the stripes even under focused measurement conditions.

In the other mode, the illumination within the system FOV is homogenized by slightly misfocusing the transmitter. This arrangement means that the spatial resolution of the measurement would be defined by the pixel count in the 2D SPAD array within the whole range of the imager. Thus, the spatial resolution can be higher but at the cost of a lower SNR. In both cases, however, 
block-based illumination can be used to improve system performance than with flood illumination when using the same average optical illumination power.

\section{Acknowledgments}

This work was supported financially by the Academy of Finland (Grant No. 317144), which is gratefully acknowledged. The contributions of Jari-Pekka Nousiainen and Mika Aikio to the design of the PCB layout and the optics of the transmitter, respectively, are gratefully acknowledged.

\section{References}

1. X. Liang et al., "Terrestrial laser scanning in forest inventories," ISPRS J. Photogramm. Remote Sens. 115, 63-77 (2016).

2. M. Vázquez-Arellano et al, "3-D imaging systems for agricultural applications—a review," Sensors 16(5), 618 (2016).

3. C. Wang, Z. Liu, and S. Chan, "Superpixel-based hand gesture recognition with Kinect depth camera," IEEE Trans. Multimedia 17(1), 29-39 (2015).

4. B. Schwarz, "Mapping the world in 3D," Nat. Photonics 4(7), 429-430 (2010).

5. V. C. Coffey, "Imaging in 3-D: killer apps coming soon to a device near you!" Opt. Photonics News 25(6), 36-43 (2014).

6. M. A. Albota et al., "Three-dimensional imaging laser radar with a photon-counting avalanche photodiode array and microchip laser," Appl. Opt. 41, 7671-7678 (2002).

7. C. Niclass et al., "Design and characterization of a CMOS 3-D image sensor based on single photon avalanche diodes," IEEE J. Solid-State Circuits 40(9), 1847-1854 (2005).

8. L. Pancheri and D. Stoppa, "Low-noise CMOS single-photon avalanche diodes with $32 \mathrm{~ns}$ dead time," in 37th Eur. Solid-State Dev. Res. Conf., p. 4 (2007).

9. G. Fouche, "Detection and false-alarm probabilities for laser radars that use Geiger-mode detectors," Appl. Opt. 42(27), 5388-5398 (2003).

10. M. Henriksson, "Detection probabilities for photon-counting avalanche photodiodes applied to a laser radar system," Appl. Opt. 44(24), 5140-5147 (2005).

11. P. Keranen and J. Kostamovaara, " $256 \times 8$ SPAD array with 256 column TDCs for a line profiling laser radar,' IEEE Trans. Circuits Syst. I 66, 4122-4133 (2019).

12. J. Kostamovaara et al., "On laser ranging based on high-speed/energy laser diode pulses and single-photon detection techniques," IEEE Photonics J. 7, 1-15 (2015).

13. J. Kostamovaara, S. Jahromi, and P. Keränen, "Temporal and spatial focusing in SPAD-based solid-state pulsed time-of-flight laser range imaging," Sensors 20(21), 5973 (2020).

14. C. Niclass et al, "A 100-m range 10-frame/s $340 \times 96$-pixel time-of-flight depth sensor in 0.18- $\mu$ m CMOS," IEEE J. Solid-State Circuits 48(2), 559-572 (2013).

15. D. Bronzi et al, "Automotive three-dimensional vision through a single-photon counting SPAD camera," IEEE Trans. Intell. Transp. Syst. 17(3), $782-795$ (2016).

16. M. Perenzoni, D. Perenzoni, and D. Stoppa, "A $64 \times 64$-pixels digital silicon photomultiplier direct TOF sensor with 100-MPhotons/s/pixel background rejection and imaging/ altimeter mode with $0.14 \%$ precision up To $6 \mathrm{~km}$ for spacecraft navigation and landing," IEEE J. Solid-State Circuits 52(1), 151-160 (2017).

17. S. W. Hutchings et al, "A reconfigurable 3-D-stacked SPAD imager with in-pixel histogramming for flash LIDAR or high-speed time-of-flight imaging," IEEE J. Solid-State Circuits 54(11), 2947-2956 (2019).

18. C. Zhang et al, "A CMOS SPAD imager with collision detection and 128 dynamically reallocating TDCs for single-photon counting and 3D time-of-flight imaging," Sensors 18(11), 4016 (2018).

19. S. Jahromi et al, "A $32 \times 128$ SPAD-257 TDC receiver IC for pulsed TOF solid-state 3-D imaging," IEEE J. Solid-State Circuits 55(7), 1960-1970 (2020).

20. U. Kabuk, “4D solid-state lidar,” in Int. SPAD Workshop ISSW, University of Edinburgh, Edinburgh (2020). 
21. A. Srowik, "256 $\times 16$ SPAD array and 16-channel ultrashort pulsed laser driver for automotive lidar," in Int. SPAD Workshop ISSW, University of Edinburgh, Edinburgh (2020).

22. B. S. Ryvkin, E. A. Avrutin, and J. Kostamovaara, "Asymmetric-waveguide laser diode for high-power optical pulse generation by gain switching," IEEE/OSA J. Lightwave Technol. 27(12), 2125-2131 (2009).

23. B. Lanz et al., "Performance improvement by a saturable absorber in gain-switched asymmetric-waveguide laser diodes," Opt. Express 21(24), 29780-29791 (2013).

24. J. Huikari et al., "High-energy picosecond pulse generation by gain switching in asymmetric waveguide structure multiple quantum well lasers," IEEE J. Sel. Top. Quantum Electron. 21(6), 1501206 (2015).

25. L.W. Hallman, J. Huikari, and J. Kostamovaara, "A high-speed/power laser transmitter for single photon imaging applications," in IEEE SENSORS, pp. 1157-1160 (2014).

26. M. Hintikka, L. Hallman, and J. Kostamovaara, "Comparison of the leading-edge timing walk in pulsed TOF laser range finding with avalanche bipolar junction transistor (BJT) and metal-oxide-semiconductor (MOS) switch based laser diode drivers," Rev. Sci. Instrum. 88, 123109 (2017).

27. D. Bimberg et al., "Gain modulation of unbiased semiconductor lasers: ultrashort pulse generation," Int. J. Electron. 60(1), 23-45 (1986).

28. P. Vasilev, Ultrafast Diode Lasers: Fundamentals and Applications, Artech House, Inc., Boston, London (1995).

Sahba S. Jahromi received her MSc (2011) and Dr. Tech. (2020) degrees in electronics from the University of Tehran (Iran) and University of Oulu (Finland), respectively. She is now working as a postdoctoral researcher with the Circuits and Systems Research Unit at the University of Oulu. Her research interests include single-photon detectors and circuits, pulsed TOF techniques, and solid-state 3D imagers.

Jussi-Pekka Jansson received his MSc (Tech) and Dr. Tech degrees in electrical engineering from the University of Oulu, Finland, in 2004 and 2012, respectively. He became an adjunct professor with the same university in 2017 . He has been a university researcher with the Circuits and Systems Research Unit, University of Oulu, since 2020. His current research interests include high-precision TDC architectures and applications related to them.

Pekka Keränen received the MSc (Tech) and DSc (Tech) degrees in electrical engineering from the University of Oulu, Finland, in 2010 and 2016, respectively. His main research interests include integrated circuit design for pulsed TOF laser radars, such as SPAD-based receiver circuits and TDCs. From 2008 to 2019, he was with the Circuits and Systems Research Unit, University of Oulu, Finland. Since 2019 he has been a senior analog IC design engineer with CoreHW, Oulu, Finland.

Eugene A. Avrutin started his research career at A.F. Ioffe Physico-Technical Institute, St. Petersburg, Russia, in 1986. Having worked at the Universities of Bath and Glasgow in the 1990s, he is a member of academic staff at the University of York, where his research interests are in theory and modeling of semiconductor optoelectronic components and subsystems.

Boris S. Ryvkin is a senior researcher at the A.F. Ioffe Physico-Technical Institute, St. Petersburg, Russia. He has conducted extensive experimental and theoretical research in the field of semiconductor physics and optoelectronics. He has been a visiting scientist and a visiting professor at several universities in the U.K., Belgium, Germany, and Finland. For the last 12 years, he has been working very closely with the University of Oulu, Finland.

Juha T. Kostamovaara received his Dr. Eng. (electrical engineering) degree from the University of Oulu, Finland, in 1987. Kostamovaara held the academy professorship position nominated by the Academy of Finland during 2006-2017. Currently, he acts as a professor (emeritus) in electronics at the University of Oulu (Circuits and Systems Research Unit), Finland. His main research interest is in the development of pulsed TOF devices, circuits, and systems for electronic and optoelectronic measurements. 